\title{
Circulating cell-free DNA in plasma of melanoma patients: Qualitative and quantitative considerations
}

\author{
Pamela Pinzani a,*, Francesca Salvianti a , Sara Zaccara ${ }^{a}$, Daniela Massi ${ }^{\text {b }}$, Vincenzo De Giorgi ${ }^{\text {c }}$ \\ Mario Pazzagli a , Claudio Orlando ${ }^{\text {a }}$ \\ a Department of Clinical Physiopathology, University of Florence, Viale Pieraccini 6, 50139 Florence, Italy \\ ${ }^{b}$ Department of Human Pathology, University of Florence, Viale G.B. Morgagni 85, 50139 Florence, Italy \\ c Department of Dermatological Sciences, University of Florence, Via della Pergola 60, Florence, Italy
}

\section{A R T I C L E I N F O}

\section{Article history:}

Received 27 May 2011

Received in revised form 15 July 2011

Accepted 25 July 2011

Available online 3 August 2011

\section{Keywords:}

Cell-free DNA

DNA integrity index

qPCR

Melanoma

\begin{abstract}
A B S T R A C T
DNA integrity in blood is an emerging biomarker in cancer. Here we report a real time PCR approach for the absolute quantification of four amplicons of $67,180,306$ and 476 bp in cutaneous melanoma. Three different integrity indexes (180/67, 306/67 and 476/67 ratios) were tested for their ability to reflect differences in plasma cell-free DNA (cfDNA) fragmentation in 79 patients affected by cutaneous melanoma and 34 healthy subjects. All the three integrity indexes showed higher values in melanoma patients in comparison with healthy subjects.

According to ROC curve analysis, the ratio $180 / 67$ is the most suitable index to be used in cancer patient selection, even if the combination of the 3 indexes gives the best performance in terms of clinical sensitivity. The most represented fragments in plasma of melanoma patients are those comprised between 181 and $307 \mathrm{bp}$, while in healthy subjects there is a prevalence of shorter fragments (67-180 bp).

In conclusion, DNA integrity indexes can be considered suitable parameters for monitoring cfDNA fragmentation in melanoma patients.
\end{abstract}

(c) 2011 Elsevier B.V. All rights reserved.

\section{Introduction}

The hypotheses on the origin of cell-free DNA (cfDNA) are still controversial and details on the exact mechanisms of release are not completely disclosed [1].

Previous studies demonstrated an increase of circulating cfDNA in different types of cancer (see Ref. [2] for a review) in comparison to healthy population. In affected patients, DNA concentration in plasma can be influenced by tumor stage, size and location [3].

However, these values may also be altered in patients with various diseases such as trauma, stroke, burns, sepsis, and autoimmune diseases, thus limiting their value for diagnosis of cancer [1].

Therefore quantitative analyses limited to cfDNA concentration cannot provide the expected clinical specificity, unless combined with qualitative alterations of DNA, such as mutations, loss of heterozigosity (LOH), microsatellite instability and epigenetic changes [3]. The detection of these biomarkers entails sophisticated techniques due to their scarcity in plasma if compared to wild type sequences and the high

\footnotetext{
Abbreviations: cfDNA, cell-free DNA; qPCR, quantitative real time PCR.

* Corresponding author at: Department of Clinical Physiopathology, University of Florence, Viale Pieraccini 6, 50139 Firenze, Italy. Tel.: + 390554271441; fax +390554271371 .

E-mail address: p.pinzani@dfc.unifi.it (P. Pinzani).
}

sensitivity requested for their detection makes these approaches challenging for immediate clinical applications.

Circulating cfDNA is released from apoptotic or necrotic cells, reflecting a differential DNA origin, as well as from living cells through a mechanism of active release [1,4]. Necrosis is common in solid malignant tumors and generates a spectrum of DNA fragments with variable size, due to random digestion by DNases. In contrast, cell death in normal blood nucleated cells occurs mostly via apoptosis that generates small and uniform DNA fragments. In support to this hypothesis, recent studies demonstrated increased DNA length in plasma from patients with breast [5] and gynecologic cancers in comparison to healthy subjects [6]. Moreover, DNA characterized by higher integrity could be evidenced in cancer of the head and neck [7], prostate [8], kidney [9,10], esophagus [11], nasopharynx [12] and colon [13]. The test is based on the hypothesis that DNA fragments in plasma of cancer patients are longer than those detectable in healthy individuals.

Nonetheless, a limited number of studies reported decreased DNA integrity in cancer patients compared to the healthy population [1416] or were unable to demonstrate a difference between cancer and non-cancer patients $[17,18]$. This could be due to distinctive features of different cancer types or to preanalytical and analytical factors [3].

Our attention was focused on the qualitative and quantitative characterization of cfDNA in patients with cutaneous melanoma.

We adopted a quantitative real time PCR (qPCR) approach based on the measurement of amplicons of increasing length to determine 
three different integrity indexes, able to reflect plasma cfDNA fragmentation.

The method was evaluated by comparing the integrity indexes in healthy subjects and melanoma patients as well as in pre and post surgical samples from the same patient.

\section{Material and methods}

\subsection{Patients}

Seventy-nine consecutive patients treated at the Department of Dermatological Sciences of University of Florence, were evaluated for plasma DNA integrity. Blood samples were collected before primary tumor resection or, for metastatic patients, before lymph node or subcutaneous metastases excision.

The group included patients undergoing surgery for in situ melanoma ( $n=12$ ) (median: 59.5 range: $39-80 \mathrm{yrs}$ ), primary invasive melanoma $(n=57)$ (median: 67 range: $23-88)$ and metastatic melanomas $(n=10)$ (median: 48.5 range: 28-94). In addition, 34 healthy subjects (median: 59 range: 29-85), who voluntarily donated their blood to be submitted to plasma DNA integrity evaluation, were chosen as the control population. The clinical pathological parameters of primary cutaneous invasive melanomas are reported in Table 1.

A subgroup of 21 patients was submitted to a second blood drawing after 15 days from surgery.

The research protocol was approved by the local ethic board and all the patients signed an informed consent.

\subsection{DNA extraction}

A $5 \mathrm{ml}$ aliquot of peripheral blood was collected in EDTA tubes, transported within $1 \mathrm{~h}$ to the laboratory and centrifuged at $4{ }^{\circ} \mathrm{C}$ for $10 \mathrm{~min}$ (1600 rcf); supernatant was recovered and centrifuged at

Table 1

Description of the clinical pathological parameters of primary cutaneous invasive melanomas $(\mathrm{n}=57)$.

\begin{tabular}{lc}
\hline Parameter & Cases, $\mathrm{n}(\%)$ \\
\hline Thickness & \\
$\leq 1 \mathrm{~mm}$ & $35(61.4)$ \\
$1.01-2.0 \mathrm{~mm}$ & $12(21.0)$ \\
$2.01-4.0 \mathrm{~mm}$ & $7(12.3)$ \\
$>4 \mathrm{~mm}$ & $3(5.3)$ \\
& \\
Level & \\
II & $12(21.0)$ \\
III & $21(36.8)$ \\
IV & $24(42.1)$ \\
Ulceration & \\
Absent & \\
Present & $47(82.5)$ \\
Anatomic site & $10(17.5)$ \\
Head and neck & \\
Trunk & \\
Extremities & $4(7.0)$ \\
Acral sites & $32(56.1)$ \\
Genital regions & $17(29.8)$ \\
Histotype & $3(5.2)$ \\
Superficial spreading melanoma & $1(1.7)$ \\
Nodular melanoma & \\
Lentigo maligna melanoma & \\
Acral-lentiginous melanoma & $48(84.2)$ \\
Regression & $6(12.8)$ \\
Pbsent & $2(3.5)$ \\
\hline & $2(3.5)$ \\
& \\
& \\
& \\
& \\
& \\
&
\end{tabular}

$14,000 \mathrm{rcf}$ for $10 \mathrm{~min}$ at $4{ }^{\circ} \mathrm{C}$. Plasma aliquots $(600 \mu \mathrm{l})$ were stored at $-80^{\circ} \mathrm{C}$ before use. DNA was extracted from $500 \mu$ of plasma, using the QIAamp DSP Virus Kit (Qiagen, Italy) and RNAse digestion to prevent RNA interference during assay reaction. Elution volume was $20 \mu \mathrm{l}$.

\subsection{Plasma DNA integrity indexes by $q P C R$}

The quantity and integrity of the cfDNA circulating in plasma was evaluated by qPCR targeting the human APP gene (Amyloid Precursor protein, chr. 21q21.2, accession NM_000484). The assays were designed in a way that the forward primer and the probe were the same for all amplicons, whereas the reverse primer varied (see Table 2 for sequences). The length of the amplicons were 67, 180, 306 and 476 bp respectively.

Absolute quantification of the shortest amplicon (67 bp) was performed in plasma samples to accurately measure the amount of free circulating DNA per ml plasma [19]. This assay was assumed to be able to measure the total amount of circulating plasma DNA, including fragments down to $67 \mathrm{bp}$ in length. Quantification of DNA concentration was obtained by interpolation on an external reference curve of genomic DNA ranging from 10 to $10^{5} \mathrm{pg} /$ reaction.

DNA extracted from a blood pool of healthy donors, measured spectrophotometrically (Nanodrop ND1000, Nanodrop, USA) and evaluated for integrity characteristics by gel electrophoresis on agarose gel was employed as the standard.

All measurements were performed from a $500 \mu \mathrm{l}$-aliquot of patient's plasma.

qPCR assays were run in the 7900HT Fast Instrument (Applied Biosystems). Thermal cycling setting was performed in order to achieve a comparable qPCR efficiency among the four different amplicons. In particular thermal conditions included a denaturation step at $95^{\circ} \mathrm{C}$ for 10 min and 50 cycles of PCR as follows: i) $15 \mathrm{~s}$ at $95^{\circ} \mathrm{C}$ and $60 \mathrm{~s}$ at $60^{\circ} \mathrm{C}$ for both 67 and $180 \mathrm{bps}$ amplicons, ii) $15 \mathrm{~s}$ at $95^{\circ} \mathrm{C}, 60 \mathrm{~s}$ at $56^{\circ} \mathrm{C}$ and $60 \mathrm{~s}$ at $72{ }^{\circ} \mathrm{C}$ for the longer amplicons of 306 and $476 \mathrm{bps}$. All the measurements were performed in triplicate on $1 \mu \mathrm{l}$ of DNA.

Each integrity index was calculated as the ratio between the absolute concentration of the longer amplicons $180 \mathrm{bp}, 306 \mathrm{bp}$ and $476 \mathrm{bp}$ and the total cf DNA amount (amplicon $67 \mathrm{bp}$ ).

Alternatively the results were used to evaluate the fraction of plasma DNA fragments with length varying from 67 to $180 \mathrm{bp}$, from 181 to $306 \mathrm{bp}$, from 307 to 476 by subtracting the absolute concentration of the longer fragment to that of the shorter one. These results were normalized on the total cfDNA amount and expressed as a percentage of it.

\subsection{Statistical analysis}

Statistical analysis was carried out using the SPSS software package 17.0 (SPSS, Chicago, USA). Statistical differences between quantitative data were evaluated by Student's $t$ test for unpaired or paired samples. A p value lower than 0.05 was considered statistically significant. All the results were reported as mean \pm standard error (mean \pm S.E.).

\section{Results}

\section{1. qPCR efficiency for size specific amplicons}

The efficiency each qPCR assay was evaluated in 5 different runs using five ten-fold dilutions of genomic DNA, ranging from $100 \mathrm{ng}$ to $10 \mathrm{pg}$. For the $67 \mathrm{bp}$ amplicon the slope $=-3.37 \pm 0.06$ (mean efficiency $=98 \%$ ) and Y-intercept $=38.25 \pm 1.8$, with coefficient of correlation always higher than 0.99 . Analogously the analysis was repeated for the other amplicons (Fig. 1) obtaining a perfect parallelism. 
Table 2

Primers and probe sequences.

\begin{tabular}{|c|c|c|c|}
\hline \multirow[t]{2}{*}{ Amplicon length } & Hydrolysis probe & Forward primer & Reverse primers \\
\hline & (5'-FAM, 3'-TAMRA) & $\left(5^{\prime}-3^{\prime}\right)$ & $\left(5^{\prime}-3^{\prime}\right)$ \\
\hline $67 \mathrm{bp}$ & ACCCCAGAGGAGCGCCACCTG & TCAGGTTGACGCCGCTGT & TTCGTAGCCGTTCTGCTGC \\
\hline 180 bp & & & TCTATAAATGGACACCGATGGGTAGT \\
\hline 306 bp & & & GAGAGATAGAATACATTACTGATGTGTGGAT \\
\hline 476 bp & & & TAAAGTAGGACTTAATTGGGTCACAAAC \\
\hline
\end{tabular}

The assays showed a sensitivity suitable to the measurement of cfDNA using a sample volume equivalent to $25 \mu \mathrm{l}$ of plasma.

\subsection{Plasma cfDNA concentration and fragment-size composition in pre- surgery melanoma and control samples}

cfDNA concentrations (mean $\pm \mathrm{SE}$; $\mathrm{ng} / \mathrm{ml}$ plasma) in plasma of healthy subjects are reported in Table 3 . The results show a decrease of cfDNA concentrations as the amplicon dimensions increase in both healthy and melanoma subjects (Fig. 2A).

Moreover, for each amplicon we found a statistically significant difference between healthy individuals and patients, with constant higher values in the melanoma group (Table 3).

Analyzing the integrity indexes $180 / 67,306 / 67$ and 476/67, invariably higher values were found in melanoma patients than in control subjects (Table 3), (Fig. 2B). Statistically significant differences can be noticed for all the considered ratios (Table 3 ).

Within the group of invasive melanomas, no significant correlation was found between each DNA integrity index and clinical-pathological parameters, such as Breslow thickness, Clark level, presence of ulceration, anatomic site, histotype and regression (data not shown).

Calculating the amount of fragments whose dimension is comprised between each amplicon sizes ( see Section 2.3), we were able to evidence that around $50 \%$ of the total cfDNA composition in healthy subjects is related to the presence of fragments of $67-180 \mathrm{bp}$ of length. On the contrary, almost $50 \%$ of DNA fragments encountered in melanoma patients before surgery were comprised between 181 and 306. Both DNA fragment categories showed concentrations significantly different in the two groups $(p<0.0001$ and $p=0.039$, respectively). On the contrary, the percentage of fragments between 307 and 476 bp (20\% in melanoma patients) was not statistically different between the two groups (Fig. 2C).

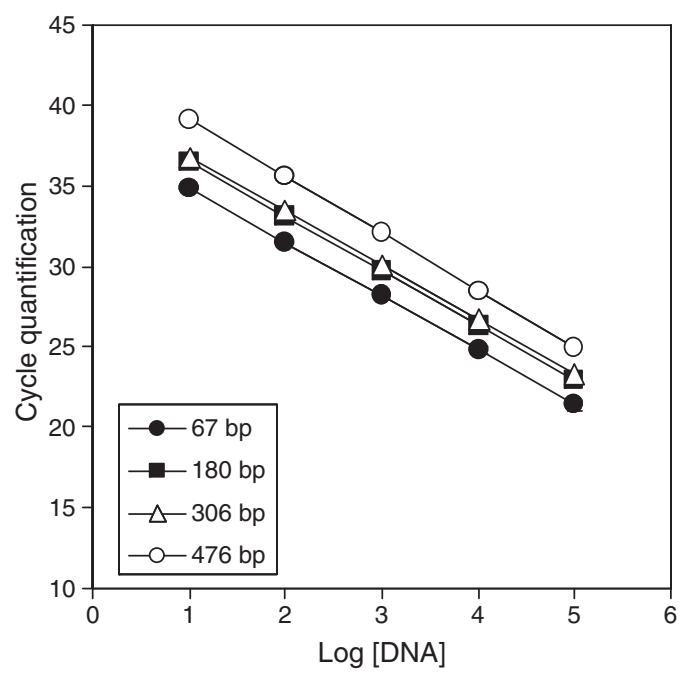

Fig. 1. Standard curve plots for the qPCR assays targeting 67, 180, 306, $476 \mathrm{bp}$ amplicons. Quantification cycle plotted versus log DNA concentration.

\subsection{Plasma DNA integrity in post surgery blood samples}

We studied total plasma DNA concentration 15 days after melanoma resection in a subgroup of 21 patients ( 2 in situ, 17 invasive and 2 metastatic melanomas).

No statistical differences were evidenced between pre-surgery $(9.6 \pm 2.5,8.6 \pm 2.7,3.0 \pm 0.5$ respectively for the 67,180 and $306 \mathrm{bp}$ amplicon) and post-surgery $(10.8 \pm 0.9,4.0 \pm 0.4,2.4 \pm 0.3$ respectively for the 67,180 and 306 bp amplicon) total cfDNA concentration, with the exception of the longest amplicon resulting $1.2 \pm 0.3$ presurgery and $0.4 \pm 0.1$ post-surgery $(p=0.025)$.

A reduction of all the considered integrity indexes could be noticed after 15 days from tumor removal $(\mathrm{p}<0.05)$. In fact, integrity index $180 / 67$ resulted $0.8 \pm 0.1$ pre-surgery and was reduced to $0.4 \pm 0.05$ after 15 days $(\mathrm{p}=0.003)$. Similarly, the index 306/67 showed a reduction of about $50 \%$ (from $0.4 \pm 0.06$ to $0.2 \pm 0.03 ; \mathrm{p}=0.011$ ) and the index $476 / 67$ was even more strongly reduced $(80 \%$ mean reduction; $\mathrm{p}=0.012$ ) changing from $0.2 \pm 0.06$ to $0.05 \pm 0.01$ (Fig. 3A).

The percentage of fragments between 67 and $180 \mathrm{bp}$ was significantly enhanced 15 days after surgery $(60.5 \pm 5.6 \%$ versus $37.0 \pm 5.8, \mathrm{p}=0.004)$. On the contrary, the percentage of fragments between 180 and $306 \mathrm{bp}$ was significantly reduced after surgery $(23.6 \pm 5.2 \%$ vs $65.7 \pm 12.5 \%, \mathrm{p}=0.016)$.

A non statistically significant decrease in the percentage of fragments between 307 and 476 bp was observed after surgery $(45.5 \pm 18.9 \%$ versus $22.5 \pm 3.3, \mathrm{p}=0.259)$ (Fig. 3B).

\subsection{Clinical sensitivity and specificity}

ROC curve analysis was used to assess the performances of our evaluation of DNA integrity in melanoma patients and controls. The area under the ROC curves for melanoma versus healthy patients, the clinical sensitivities and specificities at chosen cut-off values are reported in Table 4 for each integrity index. By evaluating the case study in terms of positivity/negativity on the basis of the chosen cutoffs, no integrity index is capable by itself to identify more than $68 \%$ of melanoma patients, while combining the 3 parameters we can reach a higher sensitivity. In fact 74/79 melanoma patients result above the reported cut-off for at least one parameter, with a total sensitivity of $93.7 \%$.

Table 3

cfDNA concentration and integrity indexes (mean $\pm \mathrm{SE}$ ) with corresponding $\mathrm{p}$ values.

\begin{tabular}{|c|c|c|c|}
\hline \multirow[t]{2}{*}{ Amplicon size } & Melanoma patients & Controls & \multirow[t]{2}{*}{ Probability $(\mathrm{P})$} \\
\hline & $(n=67)$ & $(n=34)$ & \\
\hline 67 bp & $12.4 \pm 1 . .4$ & $8.2 \pm 0.9$ & 0.015 \\
\hline 180 bp & $9.1 \pm 0.9$ & $4.4 \pm 0.7$ & $<0.001$ \\
\hline 306 bp & $2.9 \pm 0.3$ & $1.8 \pm 0.2$ & 0.001 \\
\hline 476 bp & $1.9 \pm 0.2$ & $1.0 \pm 0.2$ & 0.006 \\
\hline $180 / 67$ bp ratio & $0.8 \pm 0.05$ & $0.5 \pm 0.04$ & $<0.001$ \\
\hline 306/67 bp ratio & $0.3 \pm 0.03$ & $0.2 \pm 0.02$ & 0.008 \\
\hline $476 / 67$ bp ratio & $0.2 \pm 0.03$ & $0.1 \pm 0.01$ & 0.002 \\
\hline
\end{tabular}




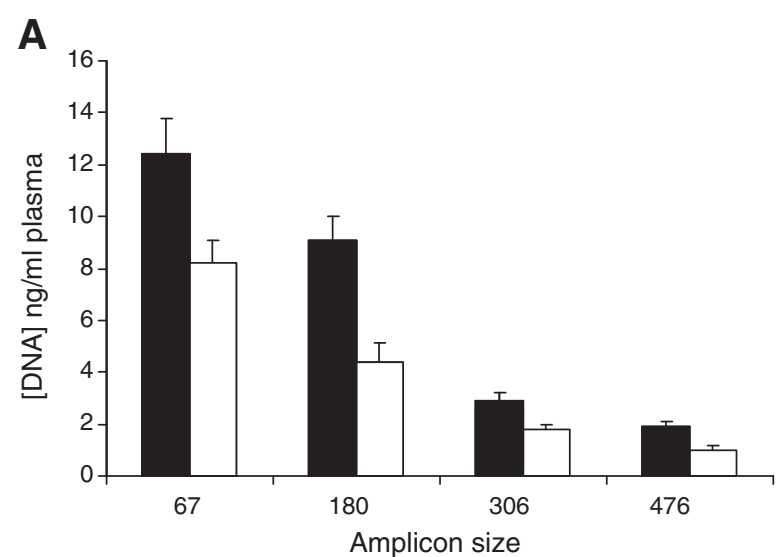

B
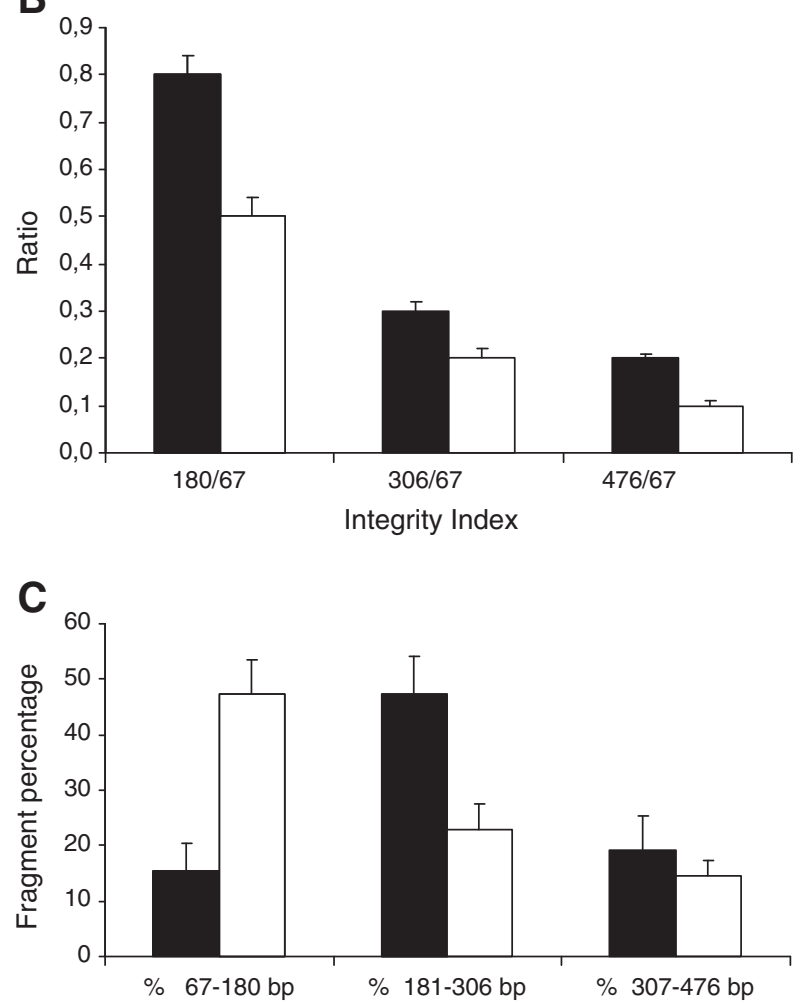

Fig. 2. cfDNA concentration (A), integrity indexes (B) and percentages of DNA fragments of a defined length range $(C)$ in melanoma patients $(n=79$, black columns) and healthy subjects ( $n=34$, white columns). Data are reported as mean \pm S.E.

\section{Discussion}

The aim of the present work was to study cfDNA integrity in cutaneous melanoma, so far not investigated about this aspect.

The study was directed to the quantitative determination of circulating DNA by means of four qPCR assays differing for the amplicon length (67, 180, 306 and 476 bp respectively).

Using this approach we confirmed the potential diagnostic ability of the cfDNA circulating in plasma to discriminate cancer patients from healthy control subjects. Melanoma patients had higher levels of cfDNA than healthy subjects (independently from amplicon length), similarly to already published results for different types of cancer $[2,3]$.

A statistically significant increase could be evidenced in melanoma patients when compared to healthy subjects for the three integrity indexes calculated in this study (180/67, 306/67 and 476/67), in agreement to already reported data. In fact, notwithstanding a great
A
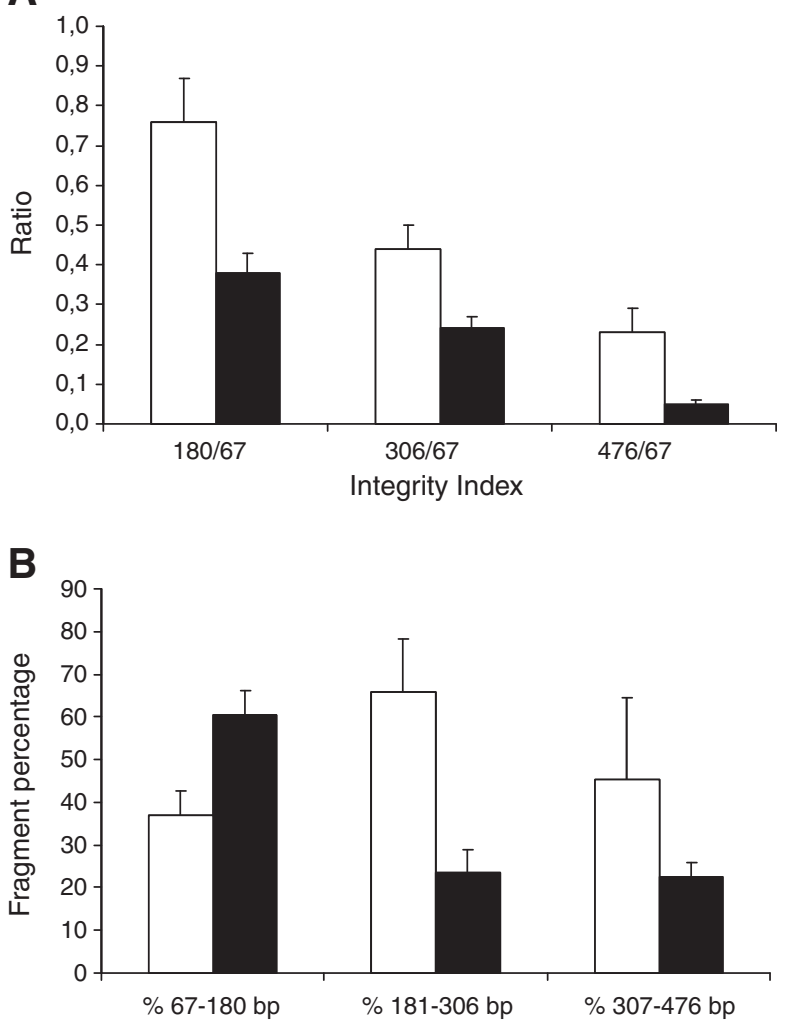

Fig. 3. (A) Integrity indexes (mean \pm S.E.) in melanoma patients $(n=21)$ pre-surgery (white columns) and 15 days after surgery (black columns). (B) Plasma cell-free DNA fragment composition. Percentages of DNA fragments of a defined length range presurgery (white columns) and 15 days after surgery (black columns).

heterogeneity in the pre analytical and analytical steps, most of the papers, based on qPCR approaches to measure DNA fragmentation in plasma, report an increase of integrity index in tumor patients in comparison to the healthy population $[5-7,11,13]$.

The construction of a ROC curve allowed the identification of the ratio $180 / 67$ as the most suitable index to be used in melanoma patient selection. Nevertheless, the combination of the 3 indexes (180/67, 306/67 and 476/67) gives the best performance in terms of clinical sensitivity.

On the basis of our results the most represented fragments in plasma of melanoma patients are those comprised between 181 and $306 \mathrm{bp}$, while in healthy subjects there is a prevalence of shorter fragments (67$180 \mathrm{bp}$ ). It is generally accepted that the $180 \mathrm{bp}$-fragment reflects apoptosis, which is the prevalent mechanism of cell death in normal cells, while necrosis, producing much longer DNA fragments, seems to occur more frequently in tumor cells [20,21].

Post-surgical melanoma samples show a higher percentage of small DNA fragments (67-180 bp) together with a lower percentage of long DNA fragments (181-306 bp) in comparison to the presurgery condition.

In conclusion, DNA integrity indexes resulted suitable parameters for monitoring cfDNA fragmentation in melanoma patients. As a perspective in melanoma patients, further studies using DNA integrity

Table 4

Area under ROC curves and clinical specificity/sensitivity for the 3 integrity indexes.

\begin{tabular}{ccccc}
\hline Integrity index & Area under the curve & Cut-off & Sensitivity & Specificity \\
\hline $180 / 67$ & 0.75 & 0.625 & $68 \%$ & $72 \%$ \\
$306 / 67$ & 0.56 & 0.175 & $68 \%$ & $40 \%$ \\
$476 / 67$ & 0.59 & 0.105 & $62 \%$ & $56 \%$ \\
\hline
\end{tabular}


index to monitor patients' outcome and the effect of therapy are advisable.

\section{Acknowledgments}

This work was funded by Istituto Toscano Tumori (ITT).

\section{References}

[1] van der Vaart M, Pretorius PJ. Circulating DNA. Its origin and fluctuation. Ann N Y Acad Sci 2008;1137:18-26.

[2] Fleischhacker M, Schmidt B. Circulating nucleic acids (CNAs) and cancer - a survey. Biochim Biophys Acta 2007;1775:181-232.

[3] Jung K, Fleischhacker M, Rabien A. Cell-free DNA in the blood as a solid tumor biomarker - a critical appraisal of the literature. Clin Chim Acta 2010;411: 1611-24.

[4] Chen Z, Fadiel A, Naftolin F, Eichenbaum KD, Xia Y. Circulation DNA biological implications for cancer metastasis and immunology. Med Hypotheses 2005;65: 956-61.

[5] Umetani N, Giuliano AE, Hiramatsu SH, et al. Prediction of breast tumor progression by integrity of free circulating DNA in serum. J Clin Oncol 2006;24: 4270-6.

[6] Wang BG, Huang HY, Chen YC, et al. Increased plasma DNA integrity in cancer patients. Cancer Res 2003;63:3966-8.

[7] Jiang WW, Zahurak M, Goldenberg D, et al. Increased plasma DNA integrity index in head and neck cancer patients. Int J Cancer 2006;119:2673-6.

[8] Hanley R, Rieger-Christ KM, Canes D, et al. DNA integrity assay a plasma-based screening tool for the detection of prostate cancer. Clin Cancer Res 2006;12: 4569-74.

[9] Gang F, Guorong L, An Z, Anne GP, Christian G, Jacques T. Prediction of clear cell renal cell carcinoma by integrity of cell-free DNA in serum. Urology 2010;75: 262-5.
[10] Hauser S, Zahalka T, Ellinger J, et al. Cell-free circulating DNA Diagnostic value in patients with renal cell cancer. Anticancer Res 2010;30:2785-9.

[11] Tomita H, Ichikawa D, Ikoma D, et al. Quantification of circulating plasma DNA fragments as tumor markers in patients with esophageal cancer. Anticancer Res 2007:27:2737-41.

[12] Chan KC, Leung SF, Yeung SW, Chan AT, Lo YM. Persistent aberrations in circulating DNA integrity after radiotherapy are associated with poor prognosis in nasopharyngeal carcinoma patients. Clin Cancer Res 2008;14:4141-5.

[13] Umetani N, Kim J, Hiramatsu S, et al. Increased integrity of free circulating DNA in sera of patients with colorectal or periampullary cancer direct quantitative PCR for ALU repeats. Clin Chem 2006;52:1062-9.

[14] Ellinger J, Bastian PJ, Ellinger N, et al. Apoptotic DNA fragments in serum of patients with muscle invasive bladder cancer a prognostic entity. Cancer Lett 2008;264:274-80.

[15] Ellinger J, Bastian PJ, Haan KI, et al. Noncancerous PTGS2 DNA fragments of apoptotic origin in sera of prostate cancer patients qualify as diagnostic and prognostic indicators. Int J Cancer 2008;122:138-43.

[16] Ellinger J, Wittkamp V, Albers P, et al. Cell-free circulating DNA: diagnostic value in patients with testicular germ cell cancer. J Urol 2009;181:363-71.

[17] Schmidt B, Weickmann S, Witt C, Fleischhacker M. Integrity of cell-free plasma DNA in patients with lung cancer and nonmalignant lung disease. Ann N Y Acad Sci 2008;1137:207-13.

[18] Holdenrieder S, Burges A, Reich O, Spelsberg FW, Stieber P. DNA integrity in plasma and serum of patients with malignant and benign diseases. Ann N Y Acad Sci 2008;1137:162-70.

[19] Lehmann U, Glöckner S, Kleeberger W, von Wasielewski HF, Kreipe H. Detection of gene amplification in archival breast cancer specimens by laser-assisted microdissection and quantitative real-time polymerase chain reaction. Am J Pathol 2000;156:1855-64.

[20] Jahr S, Hentze H, Englisch S, et al. DNA fragments in the blood plasma of cancer patients quantitations and evidence for their origin from apoptotic and necrotic cells. Cancer Res 2001;61:1659-65.

[21] Suzuki N, Kamataki A, Yamaki J, Homma Y. Characterization of circulating DNA in healthy human plasma. Clin Chim Acta 2008;387:55-8. 